\title{
Urgências em traumatismos dentários: classificação, características e procedimentos
}

\author{
Dental traumatism urgencies: classification, signs and procedures
}

Mariane Emi Sanabeํ, Lícia Bezerra Cavalcante², Cármen Regina Coldebella³, Fabio Cesar B. de Abreu-e-Lima

\section{RESUMO}

Objetivo: Discutir os aspectos das urgências odontológicas relacionadas aos traumatismos dentários, disponibilizando mais informações para médicos pediatras ou plantonistas de serviços de atendimento de urgências e emergências.

Fontes de dados: O levantamento dos dados foi realizado na base de dados Pubmed e Bireme, selecionando os artigos dos últimos 13 anos. As palavras-chave utilizadas foram: traumatismo dentário, dente decíduo e dente permanente. Os critérios de inclusão utilizados foram: artigos em inglês e português sobre incidência, prevalência e etiologia, guias de procedimentos e casos clínicos apenas de traumatismo dentário, sendo excluídos artigos de clareamento de dentes traumatizados, traumas faciais ósseos e casos clínicos de acompanhamento reduzido.

Síntese dos dados: Os dados foram descritos de forma concisa para se tornar um guia de fácil leitura e rápido acesso em relação à conduta, necessidade de atendimento imediato e correta escolha de soluções para armazenagem dos dentes e fragmentos.

Conclusões: $\mathrm{O}$ conhecimento sobre o assunto, a agilidade no tratamento de urgência e o correto encaminhamento do paciente proporcionam melhor prognóstico.

Palavras-chave: dente; traumatismo dentário; dente decíduo; dente permanente.

\section{ABSTRACT}

Objective: The aim of this literature review is to discuss the clinical aspects of dental urgencies related to dental traumatisms, providing more information for health professionals who work in emergency units, such as pediatricians or physicians on-call and nurses.

Data source: The studies were searched and selected in the Pubmed and Bireme databases, from the past 13 years. The keywords were: tooth injuries, deciduous tooth and permanent tooth. The inclusion criteria were: articles written in English and Portuguese related to incidence, prevalence, cause, guidelines and case reports of dental traumatism. Studies about dental bleaching in dental trauma, face bone trauma and reduced post-operative case reports were excluded.

Data synthesis: The data were briefly described so that it can be easily read and quickly accessible regarding procedures, urgency attention and correct choice for dental and fragment storage.

Conclusions: The knowledge on the subject, the agility to deal with urgent situations and the correct patient followup improve prognosis.

Key-words: tooth; tooth injuries; tooth, deciduous; tooth, permanent.

\footnotetext{
Instituição: Departamento de Clínica Infantil da Faculdade de Odontologia de Araraquara da Universidade Estadual Paulista (Unesp), Araraquara, SP, Brasil

'Doutora em Ciências Odontológicas pela Faculdade de Odontologia de Araraquara da Unesp, Araraquara, SP, Brasil

${ }^{2}$ Mestre em Ciências Odontológicas da Faculdade de Odontologia de Araraquara da Unesp, Araraquara, SP, Brasil

${ }^{3}$ Doutoranda em Ciências Odontológicas da Faculdade de Odontologia de Araraquara da Unesp, Araraquara, SP, Brasil

${ }^{4}$ Professor assistente Doutor da Disciplina de Odontopediatria da Faculdade de Odontologia de Araraquara da Unesp, Araraquara, SP, Brasil
}

Endereço para correspondência:

Mariane Emi Sanabe

Rua Costa Aguiar, 2324 - Ipiranga

CEP 04204-002 - São Paulo/SP

Email: emisanabe@hotmail.com

Recebido em: 6/10/08

Aprovado em: 15/2/09 


\section{Introdução}

O traumatismo dentário é uma situação de urgência, frequente nos consultórios odontopediátricos. Muitas vezes, porém, o atendimento que deveria ser imediato não é efetivamente realizado devido à falta de conhecimento de pais e responsáveis ${ }^{(1,2)}$ ou pelo fato de o primeiro atendimento ser realizado em prontos-socorros, clínicas médicas ou postos de saúde ${ }^{(3)}$. Esses fatores, associados à falta de conhecimento dos profissionais de saúde sobre traumatismos dentários, ocasionam adiamento da avaliação pelo cirurgião dentista ${ }^{(4,5)}$, afetando o seu prognóstico.

Consideram-se lesões traumáticas dentárias desde uma simples fratura em esmalte até a perda definitiva do elemento dentário. Existe uma predominância de traumatismos dentários em indivíduos do sexo masculino ${ }^{(6-8)}$, especialmente em idade escolar e em fase de crescimento ${ }^{(9)}$, como consequência de quedas, brigas ou lutas, acidentes esportivos, automobilísticos, traumatismos com objetos e maus tratos ${ }^{(6-8)}$. Situações de urgência envolvendo cabeça e pescoço frequentemente se tornam experiências dramáticas para os pais e para as crianças. As lesões que envolvem os dentes anteriores (incisivos centrais, incisivos laterais e caninos) podem resultar em efeitos desfavoráveis na função e causar sintomatologia dolorosa, afetando diretamente a autoestima, o comportamento e o sucesso pessoal, especialmente se há perda dentária permanente ${ }^{(10,11)}$. O objetivo dessa revisão é fornecer informações atualizadas sobre traumatismos dentários a médicos pediatras, enfermeiros e outros profissionais da saúde quanto à ocorrência e à conduta clínica, com o objetivo de promover o bem-estar das crianças envolvidas e de suas famílias.

O levantamento dos dados contidos nesta revisão da literatura foi realizado por meio da base de dados Pubmed e da base de dados da Biblioteca Brasileira de Odontologia (Bireme). Foram selecionados artigos publicados nos últimos 13 anos em revistas de impacto internacional na área de traumatismo dentário e na odontologia nacional. As palavras-chave utilizadas para a seleção dos artigos foram: traumatismo dentário, dente decíduo e permanente; foram incluídos artigos em inglês e português sobre prevalência e etiologia, guias de procedimentos e casos clínicos apenas de traumatismos dentários; foram excluídos os artigos de clareamento de dentes traumatizados, traumas faciais e casos clínicos de reduzido acompanhamento. A revisão de literatura deste trabalho foi redigida na forma 'conduta clínica e procedimentos', com o objetivo de se tornar um guia atualizado de fácil acesso e consulta para os profissionais da saúde.

\section{Conduta clínica e procedimentos}

A experiência do profissional é essencial para o bom andamento do tratamento após o traumatismo. É necessário, inicialmente, acalmar os pais e o paciente para que se consiga obter informações precisas durante a realização da anamnese, com vistas a estabelecer um diagnóstico preciso e confiável, por meio de perguntas simples sobre onde, como e quando ocorreu o traumatismo ${ }^{(12)}$.

A classificação e as características clínicas apresentadas nas Tabelas 1 e 2 foram adaptadas de Andreasen et a ${ }^{(9)}$ e descritas de acordo com o Guia para o Manejo de Lesões Traumáticas Dentárias ${ }^{(13,14)}$, desenvolvido em 2007 por especialistas de vários países pertencentes à Associação Internacional de Traumatologia Dental e classificadas em traumatismos dentários, do osso alveolar, luxações e avulsões dentárias.

Nas fraturas em esmalte e dentina sem exposição pulpar (Figura 1A), é dispensável o atendimento de urgência, pois a literatura mostra que o prognóstico é favorável mesmo quando o tratamento é tardio; entretanto, o atendimento odontológico é necessário para avaliação e tratamento do caso. Fratura coronária com exposição pulpar (Figuras 1B e 1C), luxação intrusiva (Figura 2C), concussão, subluxação, e traumatismo em dentes decíduos são considerados de gravidade moderada; entretanto, necessitam de atendimento imediato $^{(15)}$. Avulsão, fratura radicular (Figuras 2A e 2B) e fratura alveolar são consideradas situações agudas mais sérias e também devem receber atendimento imediato ${ }^{(14)}$.

$\mathrm{O}$ atendimento de urgência nos traumatismos dentários considerados agudos garante melhor prognóstico do caso, evitando que ocorra necrose pulpar ou perda precoce do elemento dentário. Em qualquer caso considerado agudo, o paciente deve ser imediatamente encaminhado a um cirurgião-dentista para que sejam realizados os procedimentos necessários com materiais adequados.

\section{Discussão}

É alta a frequência de traumatismos dentários em crianças e adolescentes. Indivíduos que apresentam selamento labial inadequado e que possuem protrusão da maxila maior que $5 \mathrm{~mm}$ em relação à mandíbula são mais suscetíveis à ocorrência de traumatismos dentários ${ }^{(16)}$.

Segundo Proprokowitsch et al $l^{(6)}$, em um estudo realizado na Clínica Endodôntica da Faculdade de Odontologia da Universidade de São Paulo (USP) em 1995, dentre os traumatismos dentários da dentadura permanente, a fratura 
Tabela 1 - Classificação, características e condutas em fraturas dentárias e do osso alveolar

\begin{tabular}{|c|c|c|}
\hline Classificação & Características & Condutas \\
\hline Fratura em esmalte & Perda parcial de esmalte (Figura 1A) & O elemento fraturado deve ser armazenado em soro \\
\hline $\begin{array}{l}\text { Fratura em esmalte } \\
\text { e dentina }\end{array}$ & $\begin{array}{l}\text { Perda parcial de esmalte e dentina, } \\
\text { sem envolvimento pulpar (Figura 1A) }\end{array}$ & $\begin{array}{l}\text { fisiológico para colagem (técnica de baixo custo e de } \\
\text { resultados estéticos satisfatórios) } \\
\text { feita a restauração convencional }^{(20)}\end{array}$ \\
\hline Fratura coronária & $\begin{array}{l}\text { Fratura dental envolvendo esmalte, } \\
\text { dentina e polpa (Figura 1B e } 1 \mathrm{C} \text { ) }\end{array}$ & $\begin{array}{l}\text { O atendimento de urgência deve ocorrer em até } \\
\text { três horas após o trauma, com intervenções menos } \\
\text { invasivas e melhor prognóstico. Se houver fragmentos, } \\
\text { proceder como descrito acima }\end{array}$ \\
\hline $\begin{array}{l}\text { Fratura de coroa e } \\
\text { raiz }\end{array}$ & $\begin{array}{l}\text { Fratura de esmalte, dentina, } \\
\text { cemento e polpa, podendo ocorrer } \\
\text { no sentido axial como horizontal } \\
\text { com presença de mobilidade }\end{array}$ & $\begin{array}{l}\text { Se a fratura for no sentido horizontal (Figura 2B), } \\
\text { pode-se manter o elemento radicular por meio de } \\
\text { técnicas de reposicionamento dental. É necessário o } \\
\text { tratamento endodôntico pelo risco de necrose pulpar. } \\
\text { O rápido atendimento após o trauma oferece melhor } \\
\text { prognóstico. Na fratura vertical, o único tratamento é a } \\
\text { extração do elemento dentário (Figura } 2 \mathrm{~A} \text { ) }\end{array}$ \\
\hline $\begin{array}{l}\text { Fratura da parede e } \\
\text { processo alveolar }\end{array}$ & $\begin{array}{l}\text { Fratura envolvendo a parede óssea } \\
\text { do alvéolo envolvendo ou não o } \\
\text { elemento dental }\end{array}$ & $\begin{array}{l}\text { Reposicionamento do fragmento e contenção rígida } \\
\text { ou semirrígida por quatro semanas. Necessidade de } \\
\text { acompanhamento odontológico depois de quatro, oito, } \\
24 \text { semanas a um ano }\end{array}$ \\
\hline
\end{tabular}

Tabela 2 - Classificação, características e condutas para luxação e avulsão dentária

\begin{tabular}{|c|c|c|}
\hline Classificação & Características & Condutas \\
\hline Concussão & $\begin{array}{l}\text { Lesão de tecidos de suporte sem perda } \\
\text { ou deslocamento do elemento dental }\end{array}$ & $\begin{array}{l}\text { Recomendar alimentos macios e, se necessário, } \\
\text { contenção semirrígida no caso da subluxação }\end{array}$ \\
\hline Subluxação & $\begin{array}{l}\text { Lesão de tecidos de suporte com } \\
\text { presença de hemorragia gengival }\end{array}$ & $\begin{array}{l}\text { ocorrer em mais que dois dentes, para conforto do } \\
\text { paciente }\end{array}$ \\
\hline Luxação extrusiva & $\begin{array}{l}\text { O elemento dental se desloca } \\
\text { parcialmente no sentindo axial do alvéolo } \\
\text { dental. Presença de sangramento e } \\
\text { aparência do dente alongado }\end{array}$ & $\begin{array}{l}\text { Reposicionamento do elemento dental }{ }^{(22)} \mathrm{e} \\
\text { contenção semirrígida por duas semanas }\end{array}$ \\
\hline Luxação lateral & $\begin{array}{l}\text { Deslocamento irregular do elemento } \\
\text { dental do alvéolo dental que pode } \\
\text { ser acompanhada por fratura ou } \\
\text { esmagamento do osso alveolar }\end{array}$ & $\begin{array}{l}\text { Reposicionamento do elemento dental e } \\
\text { necessidade de contenção semirrígida por quatro } \\
\text { semanas. Pode ser necessária a realização de } \\
\text { tratamento endodôntico }\end{array}$ \\
\hline Luxação intrusiva & $\begin{array}{l}\text { Deslocamento do elemento dental em } \\
\text { relação ao osso do processo alveolar. } \\
\text { Clinicamente, a coroa se apresenta } \\
\text { encurtada e existe sangramento gengival } \\
\text { (Figura 2B) }\end{array}$ & $\begin{array}{l}\text { Pode ocorrer a re-erupção dental ou então } \\
\text { necessidade de tração ortodôntica do elemento } \\
\text { dental }\end{array}$ \\
\hline Avulsão & $\begin{array}{l}\text { Perda total do elemento dental. } \\
\text { Clinicamente, o alvéolo dental fica vazio } \\
\text { ou preenchido com coágulo sanguíneo } \\
\text { (Figura 3) }\end{array}$ & $\begin{array}{l}\text { O elemento dental deve ser armazenado } \\
\text { imediatamente em leite gelado }\left(4^{\circ} \mathrm{C}\right) \text { para } \\
\text { melhor conservação dos ligamentos }{ }^{(23)} \text {. Também, } \\
\text { podem ser usados o soro fisiológico e a saliva. } \\
\text { Se reimplantado em menos de } 60 \text { minutos, o } \\
\text { prognóstico é favorável; porém, se houver demora } \\
\text { ou se o dente for mantido seco ou em soluções } \\
\text { não indicadas, o prognóstico é desfavorável, } \\
\text { levando à perda permanente }\end{array}$ \\
\hline
\end{tabular}



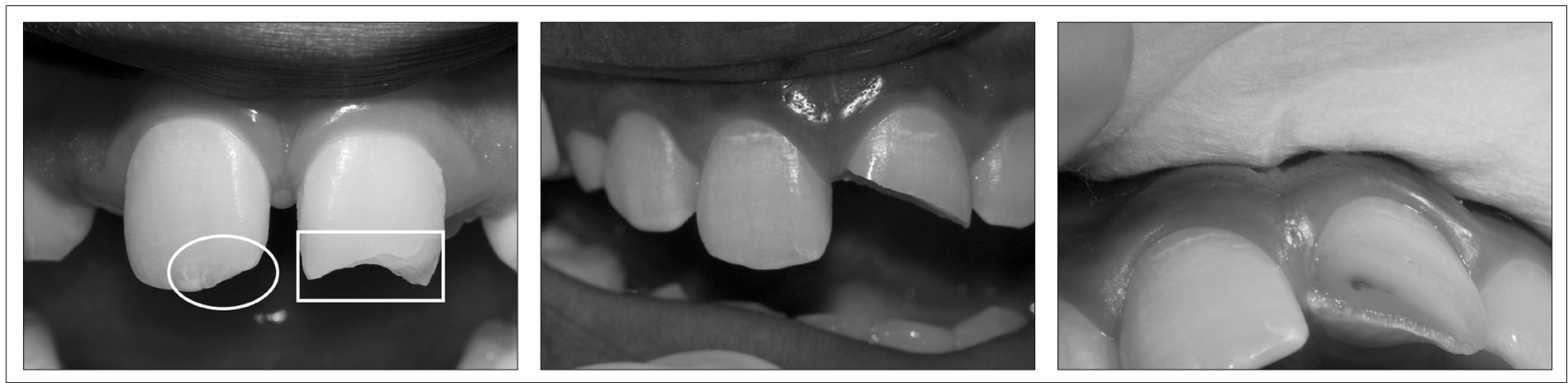

Figura 1 - Trauma em esmalte (círculo) e esmalte e dentina (retângulo) (A); fratura coronária envolvendo esmalte, dentina e polpa (B); exposição pulpar (C).
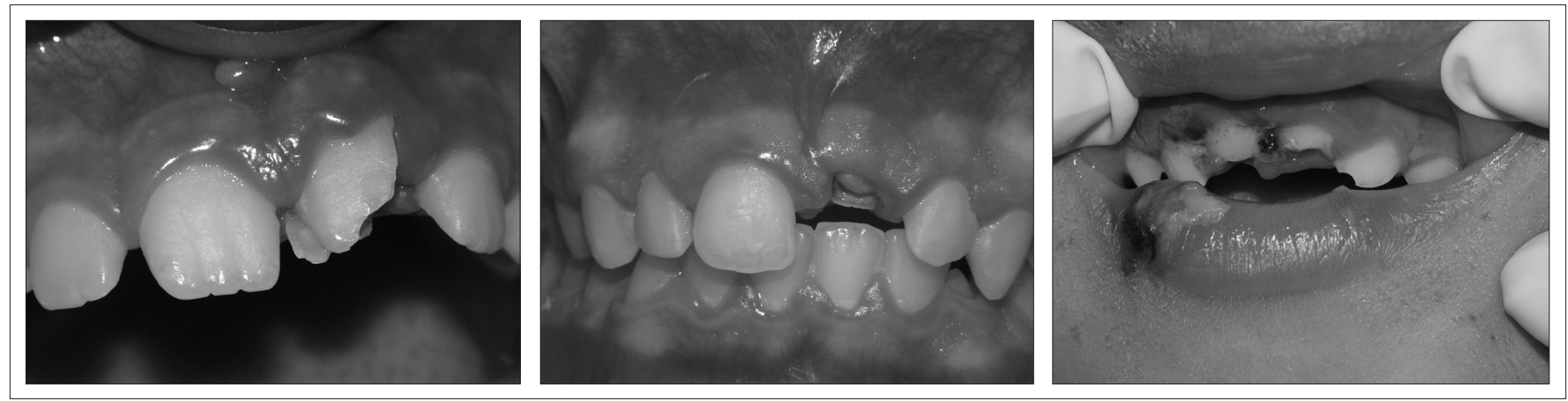

Figura 2 - Fratura coronária e radicular vertical (A); fratura radicular horizontal (B); luxação intrusiva (C).

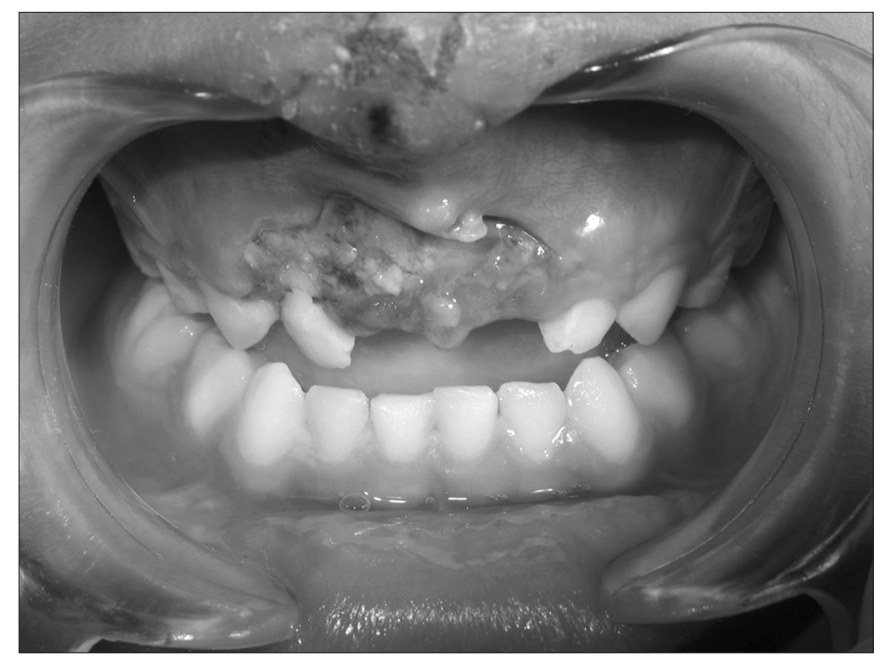

Figura 3 - Avulsão dentária.

coronária sem exposição pulpar ocorreu com maior frequência (23\%), seguido de avulsão dental (21\%), subluxação (12\%) e fratura radicular $(9 \%)$. Concussão, fratura coronária com exposição pulpar, luxação intrusiva, extrusiva, lateral e fratura de esmalte somaram os $35 \%$ restantes, com maior prevalência no sexo masculino (66\%) e faixa etária de 7 a 10 anos (40\%). Os acidentes ocorreram em sua maioria dentro de casa (35\%), seguido por queda de bicicletas (18\%), acidentes automobilísticos (16\%), queda de escada (9\%), além de brigas ou agressões, prática de esportes, acidentes com corpos estranhos e quedas na piscina em menor proporção. As consequências dos traumatismos dentários em dentes permanentes podem ser: alteração de cor, mobilidade, necrose pulpar, reabsorções ósseas e dentárias - se não tratado adequadamente -, o que pode ser ocasionada pela falta de tratamento imediato ou pelo prognóstico individual do caso.

Em estudo realizado na Clínica de Urgência Odontopediátrica da Faculdade de Odontologia da Universidade Federal do Rio Grande do Sul (UFRGS) por Porto et al ${ }^{(8)}$, os traumatismos em dentes decíduos ocorreram nos tecidos de sustentação em $79 \%$ dos casos, luxação lateral em $28 \%$, subluxação em 14\%, avulsão em $11 \%$, seguido de luxação extrusiva, concussão e fratura de alvéolo. A prevalência do traumatismo dentário foi de $20 \%$ no estudo de Porto et al ${ }^{(8)}$ e $37 \%$ no de Granville-Garcia et al ${ }^{(2)}$ em pré-escolares de Recife (PE). A faixa etária dos 2 aos 4 anos foi a mais prevalente em ambos os estudos; entretanto, nessa faixa etária, não houve diferença entre o sexo masculino e feminino. A maior ocorrência de fraturas nos tecidos de sustentação se deve ao fato de que o osso alveolar possui maior resiliência em relação ao osso alveolar permanente ${ }^{(9)}$. Como consequência das agressões a dentes decíduos, principalmente da luxação intrusiva, pode haver hipoplasia de esmalte, interrupção da formação dental, defeitos de textura, alteração no posiciona- 
mento ou erupção, má formação da coroa e raiz e distúrbios na mineralização ${ }^{(17)}$.

A higiene bucal com escovas dentárias macias e a limpeza com solução de clorexidina a $0,1 \%$ é necessária após o trauma dental para prevenir o acúmulo do biofilme dental e melhorar o prognóstico no trauma dentário ${ }^{(13,14)}$.

A conduta e procedimentos descritos neste texto tiveram como objetivo aumentar o conhecimento de médicos pediatras, enfermeiros e auxiliares sobre os traumatismos dentários e melhorar a qualidade da orientação aos pais ou responsáveis diante dessas situações ${ }^{(1,3,18,19)}$.

A negligência em relação ao tratamento odontológico após o traumatismo dentário pode ter como consequência alteração de cor, mobilidade, alteração de posição na arcada dentária, sintomatologia dolorosa, sensibilidade, reabsorções radiculares ou óssea, necrose e perda do ele-

\section{Referências bibliográficas}

1. Campos MI, Henriques KA, Campos CN. Nível de informação sobre a conduta de urgência frente ao traumatismo dental com avulsão. Pesq Bras Odontoped Clin Integr 2006;6:155-9.

2. Granville-Garcia AF, Menezes VA, Lira PI. Prevalence and sociodemographic factors associated with dental trauma in prescholers. Odontol Clin-Cient 2006;5:57-64.

3. Panzarini SR, Saad Neto M, Sonoda CK, Poi WR, Carvalho AC. Avulsões dentárias em pacientes jovens e adultos na região de Araçatuba. Rev Assoc Paul Cir Dent 2003;57:27-31.

4. Hamilton FA, Hill FJ, Mackie IC. Investigation of lay knowledge of the management of avulsed permanent incisors. Endod Dent Traumatol 1997;13:19-23.

5. Poi WR, Salineiro SL, Miziara FV, Miziara EV.A educação como forma de favorecer o prognóstico do reimplante dental. Rev Assoc Paul Cirur Dent 1999;53:474-9.

6. Prokopowitsch I, MouraAA, Davidowicz H. Fatores etiológicos e predisposição dos traumatismos dentais em pacientes tratados na clínica endodôntica da Faculdade de Odontologia da Universidade de São Paulo. RPG 1995;2:87-94.

7. Martins EL, Torriani MA, Romano AR. Estudo epidemiológico de traumatismos dos tecidos moles da face de pacientes pediátricos. J Bras Odontopediatr Odontol Bebê 2002;5:223-9.

8. Porto RB, Freitas JS, Cruz MR, Bressani AE, Barata JS, Araújo FB. Prevalence of dento-alveolar traumatisms in the urgency pediatric dental clinic of FO. UFRGS. Rev Fac Odontol Porto Alegre 2003;44:52-6.

9. Andreasen JO, Andreasen FM, Bakland LK, Flores MT. Traumatic dental injuries: a Manual. 2nd ed. Oxford: Blackwell; 2003.

10. Damasceno LM, Marassi CS, Ramos ME, Souza IP. Alterações no comportamento infantil decorrente da perda de dentes anteriores: relato de caso. Rev Bras Odontol 2002;59:193-6.

11. Vieira MV. O trauma dental não mata, porém marca! Vamos prevenir? Rev Bras Odontol 2003;60:294-5 mento dental, os quais podem acarretar dificuldades de convívio social, baixa autoestima das crianças e problemas de relacionamentos futuros, principalmente pela ausência do elemento dentário ${ }^{(10,11)}$.

Os dados apresentados refletem a necessidade de os profissionais da saúde estarem aptos a reconhecer e informar os pais e/ou responsáveis sobre a melhor conduta a ser adotada diante de um trauma dentário.

\section{Conclusão}

A ocorrência de traumatismo dentário é frequente em crianças e adolescentes, e o primeiro atendimento ao paciente, a conduta correta frente ao trauma e a agilidade para encaminhar o caso ao especialista são de extrema importância para o prognóstico.

12. Wilson CF. Management of trauma to primary and developing teeth. Dent Clin North Am 1995;39:133-67.

13. Flores MT, Andersson L, Andreasen JO, Bankland LK, Malmgren B, Barnett F et al. Guidelines for the management of traumatic dental injuries. I. Fractures and luxations of permanent teeth. Dent Traumatol 2007;23:66-71.

14. Flores MT, Andersson L, Andreasen JO, Bankland LK, Malmgren B, Barnett $F$ et al. Guidelines for the management of traumatic dental injuries. II. Avulsion of permanent teeth. Dent Traumatol 2007;23:130-6.

15. Andreasen JO, Andreasen FM, Andersson L. Textbook and color atlas of traumatic injuries to the teeth. 4th ed. Oxford: Blackwell; 2007.

16. Soriano EP, Caldas Jr AF, Góes PS. Risk factors related to traumatic dental injuries in Brazilian schoolchildren. Dent Traumatol 2004;20:246-50.

17. Campos JA, Zuanon AC, Pansani CA. Consequences of dental trauma in primary dentition to permanent dentition: a literature review. Robrac 2001;10:26-7.

18. Blakytny C, Surbuts C, Thomas A, Hunter ML. Avulsed permanent incisors: knowledge and attitudes of primary school teachers with regard to emergency management. Int J Pediatr Dent 2001;11:327-32.

19. Al-Jundi SH, Al-Waeili H, Khairalah K. Knowledge and attitude of Jordanian school health teachers with regards to emergency management of dental trauma. Dent Traumatol 2005;21:183-7.

20. Ferreira RA. Impacto radical. Rev Assoc Paul Cir Dent 1998;52:265-71.

21. Zuza EP, Silva AP, Vanzato JW, Toledo BE. Fratura radicular horizontal: relato de um caso com seis anos de proservação. J Bras Clin Estet Odontol 2000;4:21-3.

22. Silva AC, Santos RL, Aguiar CM. Procedimentos clínicos em traumas dentários J Bras Endod 2003;4:169-74.

23. Blomlöf L, Lindskog S, Andersson L, Hedström KG, Hammarström L. Storage of experimentally avulsed teeth in milk prior replantation. J Dent Res 1983;62:912-6. 